$12 / 5 / 30-95950$

UCRL-ID- 116849

\title{
A Solidification Constitutive Model For NIKE2D and NIKE3D
}

\author{
Peter J. Raboin
}

March 17, 1994

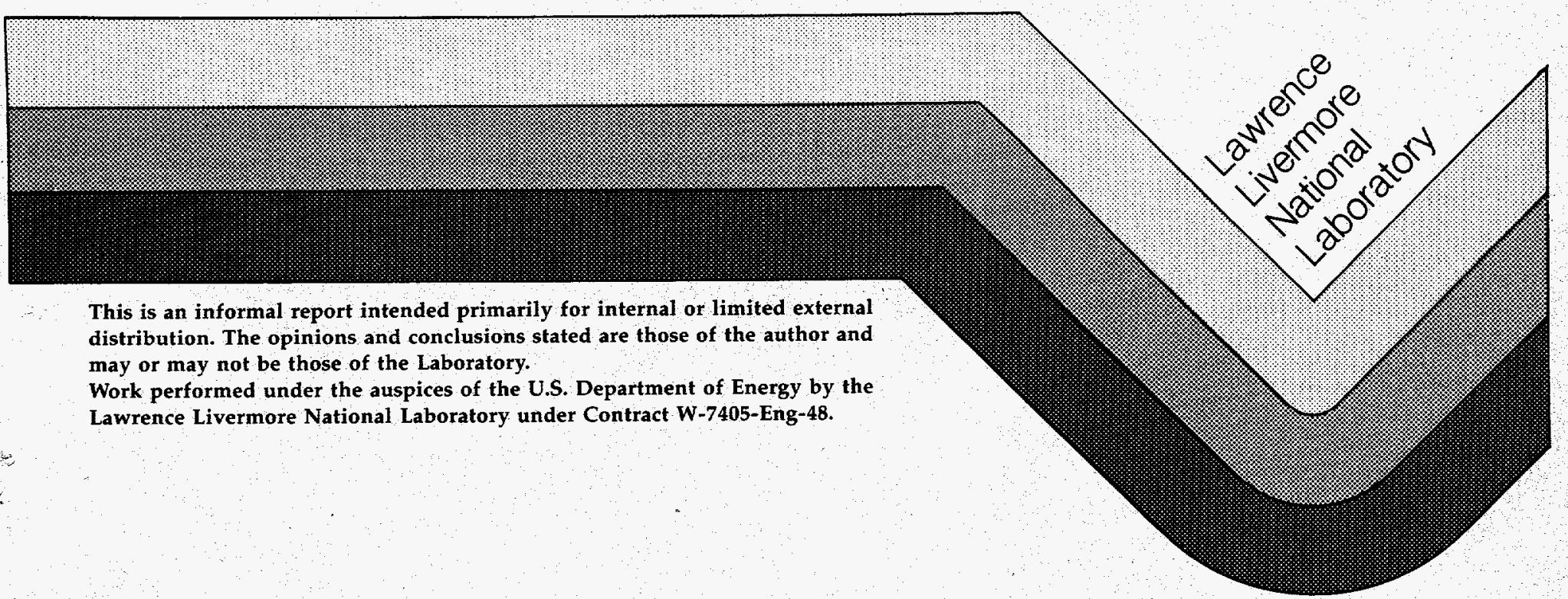




\section{DISCLAIMER}

This document was prepared as an account of work sponsored by an agency of the United States Government. Neither the United States Government nor the University of California nor any of their employees, makes any warranty, express or implied, or assumes any legal liability or responsibility for the accuracy, completeness, or usefulness of any information, apparatus, product, or process disclosed, or represents that its use would not infringe privately owned rights. Reference herein to any specific commercial products, process, or service by trade name, trademark, manufacturer, or otherwise, does not necessarily constitute or imply its endorsement, recommendation, or favoring by the United States Government or the University of California. The views and opinions of authors expressed herein do not necessarily state or reflect those of the United States Government or the University of California, and shall not be used for advertising or product endorsement purposes.

This report has been reproduced directly from the best available copy.

Available to DOE and DOE contractors from the Office of Scientific and Technical Information P.O. Box 62, Oak Ridge, TN 37831

Prices available from (615) 576-8401, FTS 626-8401

Available to the public from the

National Technical Information Service

US. Department of Commerce

5285 Port Royal Rd.

Springfield, VA 22161 


\section{DISCLAIMER}

Portions of this document may be illegible in electronic image products. Images are produced from the best available original document. 
September 20, 1993

To: B. Kornblum, B. Maker and A. Shapiro,

From: Peter Raboin

Subject: Solidification Constitutive Modeling Update

Art,

Here is my year end write-up of constitutive model development. This write-up contains some theory and lists my plans for next year (see Table 4). This memo is missing an example problems section, but this will take some additional work on my part. I want to do the $2 D$ cup analysis using rate dependent and pressure cut-off features. This memo will be changed to a UCRL in November some time. I still need to pursue some references.

\section{SUMMARY}

This memo updates the current status of a solidification material model development which has been underway for more than a year. Significant modeling goals such as predicting cut-off stresses, thermo-elasto-plasticity, strain rate dependent plasticity and dynamic recovery have been completed. The model is called SOLMAT for solidification material model, and while developed for NIKE2D, it has already been implemented in NIKE3D and NITO3D by B. Maker. This memo details the future development strategy of SOLMAT including liquid and solid constitutive improvements, coupling of deviatoric and dilatational deformation and a plan to switch between constitutive theories. It explains some of the difficulties associated solidification modeling and proposes two experiments to measure properties for using SOLMAT. Due to the sensitive nature of these plans in relation to programmatic and CRADA concerns, this memo should be treated as confidential document. 


\section{INTRODUCTION}

The solidification material model development started more than 2 years ago with test problems which scoped out the possibility of using Thermo-Elasto-Plastic model \#4 in NIKE2D to model the liquid-solid behavior of a cup of cooling Aluminum. This work eventually helped to motivate coupled code developments in PALM2D, CAST2D and NITO3D. But there were errors in the original material modeling predictions which led to unrealistic tensile stresses during cooldown. So last year an effort was started to improve model \#4. This work benefits the LLNN programmatic and CRADA casting projects.

The objective of this memo is to provide an update on the constitutive material model development for predicting the liquid-solid mechanical behavior of metals. The model development started from a solid elastoplastic constitutive rule and advanced to include pressure cut-off, strain rate dependencies and dynamic softening. At this date, the model is being evaluated with example problems to test its capabilities, robustness, and speed. In this memo, the constitutive modeling goals are stated, the current model status is reviewed, and then a future modeling development plan is presented.

\section{SOLIDIFICATION MODELING GOALS}

The first priority in developing a solidification constitutive tool from an existing solid only material model is to eliminate erroneous physical behaviors. Then, more accurate physical phenomena are added to better predict known behaviors. This development is tempered by the scarcity of information and understanding concerning the fluid-mechanical behavior of mushy metals: those above $80 \%$ of the melt temperature $T_{m}$. A modest attempt is made with this model to limit its theoretical complexity and to avoid a proliferation of material parameters. The strategy employed here is to predict the extremes of liquid and solid behavior and attempt a modeling transition.

Two glaring errors occur when liquid behavior is modeled with a simple elasto-plastic constitutive model. First, unrealistic mean tensile 
stress states may accrue. Second, deviatoric deformation can accumulate as an effective plastic strain in a liquid state and then this straining may falsely lead to an apparent flow strength increased at lower temperatures. Other errors concern the lack of strain rate dependence and the implementation of Newtonian flow behavior in a Lagrangian framework.

Eliminating mean tensile stresses at liquid temperatures is accomplished with a temperature dependent tensile stress cut-off curve. When the mean stress exceeds the tensile stress cut-off value, a normal void strain increment is generated to decrease the mean stress to the cut-off level. Thus, void strains are created and evolved to satisfy tensile stress cut-off constraints. Void strains decrease with a compressive stress cut-off mechanism. In this situation, when void strains are greater than zero and mean stresses exceed the compressive stress cut-off value, a hydrostatic void strain decrement is generated to decrease the mean stress to the cut-off level. When the void strain is zero, then the compressive stress cut-off mechanism is shut off, and the mean stress may reach any compressive level.

As a practical example, consider the diagram in Figure 1 showing tensile and compressive cut-off behavior. In the first loading sequence (points A to B) the mean stress increases proportionately with the Bulk modulus $K$ and the hydrostatic strain (Equation 1 ). When the mean stress $\bar{\sigma}$ reaches the tensile stress cut-off value $\sigma^{c o}$, then the void strain $\varepsilon^{v}$ increases with the total normal strain $\varepsilon_{k k}$ as the elastic normal strain $\varepsilon_{k k}^{e}$ remains constant (Equation 2). The void strain is calculated from Equations 1 and 2 in Equation 3. Upon reverse loading (points $B$ to $C$ ), the process is reversed until the void strain is zero. Thereafter Equation 1 applies for all further compressive straining.

$$
\begin{aligned}
\bar{\sigma} & =\sigma_{k k} / 3=K \varepsilon_{k k}^{e} \\
\varepsilon_{k k} & =\varepsilon_{k k}^{e}+\varepsilon^{v} \\
\varepsilon^{v} & =\varepsilon_{k k}-\sigma^{c o} / K
\end{aligned}
$$




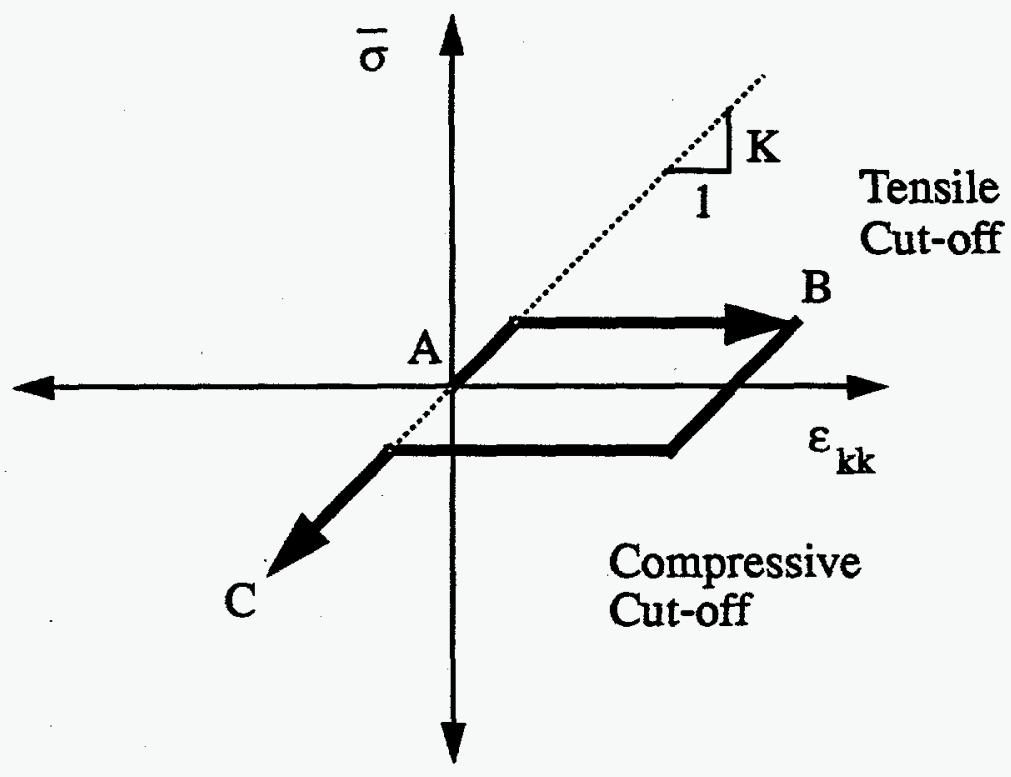

Figure 1. Diagram of tensile and compressive cut-off behavior.

The physical purpose of a cut-off stress is to mimic the presence of vapor voids in liquids. A liquid forms void spaces (where the void pressure is nearly equivalent to the liquid vapor pressure) when there is insufficient mass to fill a confined volume. The void strain relates the total void volume $V^{v}$ to the confined volume $V$ (Equation 4). Thus, the practical effect of a cut-off pressure is to nucleate or create voids through a solidification shrinkage mechanism. Without additional microstructural calculations, void strain knowledge alone is insufficient to predict the void density $\mathrm{N}^{v}$ and average void volume $\overline{\mathrm{V}}^{v}$ (Equation 5).

$$
\begin{aligned}
& \varepsilon^{v}=\frac{V^{v}}{V} \\
& \varepsilon^{v}=N^{v} \bar{V}^{v}
\end{aligned}
$$


Satisfying the goal of properly treating accumulated plastic strains is accomplished by avoiding strain hardening behavior. Avoiding the issue is an unsatisfactory approach, but for this stage of constitutive model development assuming perfect plasticity is appropriate for reasons of numerical testing and for lacking any physical data to substantiate a strain hardening assumption. All of the constitutive theories considered thus far model perfect plasticity, but it is up to user to select material parameters which simulate perfect plasticity.

The constitutive modeling selection involves four competing issues. First is what constitutive model is appropriate for modeling liquid behavior. Second, what is a good solid constitutive theory. Third, how shall the deviatoric and dilatational deformations relate to each other; void strains affect flow strength. Lastly, should the transition from liquid to solid behavior involve a switch in mechanisms or just changes in temperature dependent material parameters. Because this is a gradual model development, it starts with a solid elasto-plastic constitutive theory, adds a cut-off stress constitutive mechanism for dilatational liquid modeling, separates the deviatoric and dilatational constitutive behavior and uses temperature dependent parameters to switch from liquid to solid behavior in a single constitutive mechanism. More sophisticated solid constitutive theories have been added to this material model, and rather than eliminate earlier developments, these latter models are numbered and selectable. At the present time there are three solid constitutive theories implemented.

The first constitutive mechanism implemented is Model 0 in Equation 6 . The material flow strength $\sigma_{f}$ is defined by the addition of a Yield Strength $\sigma_{y}$ and a Hardening Modulus $H$ multiplied by the accumulated plastic strain $\bar{\varepsilon}$. This model lacks a strain rate dependence, but if the yield strength at very high temperatures is kept small (1-10 psi), then rate effects are not as important, since the strength magnitudes are small. The largest errors occur at lower temperatures where rate effects cause larger differences in strength predictions.

$$
\sigma_{f}=\sigma_{y}+H \bar{\varepsilon}^{p}
$$


Model 1 is the second solid constitutive theory (Equation 7), and it has a flow strength which depends on the deviatoric stain rate $\dot{\varepsilon}^{\prime}$ raised to a power $m$ (the strain sensitivity). By assuming the deviatoric strain rate is equivalent to the plastic strain rate (the proper rate variable), this implementation is faster and more robust. The flow strength is proportional to a Strength Coefficient $A$ and a Strength parameter $S$. The Strength parameter evolves according to Equation 8, with the rate proportional to $B$ and the plastic strain rate $\dot{\varepsilon}^{p}$. This model nearly predicts Newtonian viscous flow, except that it includes elastic stress components. For the low stress magnitudes associated with fluid behavior, compared to solid structures, the elasticity error is not large.

$$
\begin{aligned}
& \sigma_{f}=\| S \underbrace{\left(\dot{\varepsilon}^{\prime}\right)^{m}}_{A} \\
& \dot{S}=B\left(\dot{\varepsilon}^{p}\right)^{n}
\end{aligned}
$$

To model perfect plasticity with Model $1, B$ should be 0 , and for linear strain hardening make $B$ nonzero and $n$ equal to 1 . When modeling fluid behavior, $m$ equals 1 . During cooldown $m$ varies from 1.0 in the liquid state down to 0.02 to 0.2 at room temperature.

With Model 1, the Strength parameter $S$ is initialized at the analysis start and then may increase with subsequent deformation. The value of $S$ may only increase in Model 1, but for Model 2, $S$ can increase and decrease. In some instances of isothermal hardening, the flow strength increases to a maximum value where the rate of strain hardening equals the rate of dynamic relaxation. In some instances of plastic deformation, the strength decreases in a process called dynamic relaxation. At the melt temperature, all of the accumulated strength is eliminated.

The modeling of dynamic relaxation is included in Model 2 with Equations 9-11. The flow strength equation from Model 1 is unchanged but the Strength parameter is now the sum of a temperature dependent Strength Constant $S^{\circ}$ and a Strengthening Coefficient $S_{H}$, which is initially zero. The evolution of $S_{H}$ occurs through the sum of hardening and dynamic relaxation 
terms respectively. Note that the relaxation term is negative and it depends on the magnitude of $S_{H}$, so this term can drive $\dot{S}_{H}$ and $S_{H}$ to zero.

$$
\begin{aligned}
\sigma_{f} & =A S\left(\dot{\varepsilon}^{p}\right)^{m} \\
S & =S^{\circ}+S_{H} \\
\dot{S}_{H} & =k_{1}\left(\dot{\varepsilon}^{p}\right)^{n_{1}}-k_{2}\left(\dot{\varepsilon}^{p}\right)^{n_{2}}\left(S_{H}\right)^{n_{3}}
\end{aligned}
$$

The purpose of the Strength Constant $S^{\circ}$ is to vary the initial Strength Parameter value for a wide variety of temperatures without it being affected by dynamic relaxation. For instance, without $S^{\circ}$ dynamic relaxation could drive $S$ to zero at the melt temperature and then after stress free cooling to room temperature would lead to a faulty prediction of zero flow strength.

An important drawback to these three models concern those situations where the strengthening parameter increases. For most cooldown conditions, an increasing flow strength due to deformation hardening is both reasonable and expected. When a material is strengthened and then raised to high temperatures which cause annealing or melting, then whatever strengthening has occurred is dissipated. The dynamic softening mechanism described in Equation 11 requires plastic deformation to reduce the Strength variable. Softening may occur with no deformation, so a static softening mechanism is needed.

The transition in liquid-solid constitutive behavior is accomplished primarily through temperature dependent material parameters. Nearly every parameter (thermal expansion, elastic moduli and flow strength coefficients) is temperate dependent and defined with temperature-value data curves (load curves). The all important liquid-solid density changes associated with phase transformations are modeled with thermal expansions and contractions derived from the secant coefficients of thermal expansion (CTE's). The main 
advantage to these load curves is that there are no arbitrary restrictions on the quantity of temperature data pairs ( 8 for typical NIKE models).

The solidification modeling goals for this first year were to resolve the glaring errors which cause unrealistic mean stress predictions in the liquid regime, and to implement this in a solid thermo-elastic plastic material model which could predict perfect plasticity. This has been achieved, and more sophisticated strain rate behaviors have been added to the solid constitutive theories. Modeling exercise, data and experience is needed with this model before it should be publicly released.

\section{SOLIDIFICATION MODEL STATUS}

In this memo section the current implementation status, execution instructions and example problems are discussed. As previously mentioned in this memo, this is an ongoing development project, so both the implementation status and execution instructions are changing. This is another reason for postponing the open publication of this work.

For convenience sake, this material modeled is hereafter nicknamed SOLMAT for SOLidification MATerial model. It is being developed in a private version of NIKE2D. It is not yet in the 2D coupled codes. An earlier version of model 0 is in NIKE3D and NITO3D and is undergoing debugging.

SOLMAT is in a fixed version of NIKE2D, so recent improvements in NIKE2D are missing. A material slot, with SOLMAT requirements of 2 history variables, temperature dependence, and access to load curve information needs to be added to the public NIKE2D to facilitate upward compatibility. The SOLMAT in NITO3D is being developed in the public version of NIKE3D so improvements in SOLMAT or NIKE3D are automatically transferrable. SOLMAT is material model \#16 in NIKE3D.

The SOLMAT input format is patterned after Model \#18 in NIKE2D, Thermal-Elastoplastic Quench. It uses temperature load curves for just about all of its material parameters. Tables 1-3 detail the input format for the three solid constitutive models predicted with SOLMAT. Cards 1 and 2 for SOLMAT are the same default cards expected of all material models. The slideline Young's modulus $E$ and Poisson ratio $v 2$ are duplicated on Cards 4 and 
5 , because those are the input deck locations where NIKE2D Model \#4 expects to find that same information. This is likely to change in future version, despite the fact that the load curve numbers are integer quantities, their placement is in real data fields. Only the data on Card 6 is different for the three models. SOLMAT allows the initial void strain to be nonzero as a way of initializing the deformation behavior of previously solidified material.

Table 1: SOLMAT data format for Flow Strength Model Type 0

\begin{tabular}{|c|c|l|c|}
\hline Columns & Card & \multicolumn{1}{|c|}{ Quantity } & Format \\
\hline \hline $1-10$ & 3 & Young's Modulus Load Curve \# & E10.0 \\
\hline $11-20$ & & Poisson Ratio Load Curve \# & E10.0 \\
\hline $21-30$ & & Coefficient of Thermal Expansion Load Curve \# & E10.0 \\
\hline $31-40$ & & Tensile Stress Cut-off Load Curve \# & E10.0 \\
\hline $41-50$ & & Compress Stress Cut-off Load Curve \# & E10.0 \\
\hline $51-60$ & & Flow Strength Model Type (0) & E10.0 \\
\hline $61-70$ & & Initial Void Strain & E10.0 \\
\hline $1-10$ & 4 & Young's Modulus for Slideline Calculations & E10.0 \\
\hline $1-10$ & 5 & Poisson Ratio for Slideline Calculations & E10.0 \\
\hline $1-10$ & 6 & Yield Strength Load Curve \# & E10.0 \\
\hline $11-20$ & & Strengthening Modulus Load Curve \# & E10.0 \\
\hline
\end{tabular}


Table 2: SOLMAT data format for Flow Strength Model Type 1

\begin{tabular}{|c|c|l|c|}
\hline Columns & Card & \multicolumn{1}{|c|}{ Quantity } & Format \\
\hline \hline $1-10$ & 3 & Young's Modulus Load Curve \# & E10.0 \\
\hline $11-20$ & & Poisson Ratio Load Curve \# & E10.0 \\
\hline $21-30$ & & Coefficient of Thermal Expansion Load Curve \# & E10.0 \\
\hline $31-40$ & & Tensile Stress Cut-off Load Curve \# & E10.0 \\
\hline $41-50$ & & Compress Stress Cut-off Load Curve \# & E10.0 \\
\hline $51-60$ & & Flow Strength Model Type (1) & E10.0 \\
\hline $61-70$ & & Initial Void Strain & E10.0 \\
\hline $1-10$ & 4 & Young's Modulus for Slideline Calculations & E10.0 \\
\hline $1-10$ & 5 & Poisson Ratio for Slideline Calculations & E10.0 \\
\hline $1-10$ & 6 & Initial Strength $S$ & E10.0 \\
\hline $11-20$ & & Strength Coefficient $(A)$ Load Curve \# & E10.0 \\
\hline $21-30$ & & Strain Rate Sensitivity $(m)$ Load Curve \# & E10.0 \\
\hline $31-40$ & & Strengthening Coefficient $(B)$ Load Curve \# & E10.0 \\
\hline $41-50$ & & Strengthening Exponent $(n)$ Load Curve \# & E10.0 \\
\hline
\end{tabular}


Table 3: SOLMAT data format for Flow Strength Model Type 2

\begin{tabular}{|l|l|l|c|}
\hline Columns & Card & & Quantity \\
\hline \hline $1-10$ & 3 & Young's Modulus Load Curve \# & E10.0 \\
\hline $11-20$ & & Poisson Ratio Load Curve \# & E10.0 \\
\hline $21-30$ & & Coefficient of Thermal Expansion Load Curve \# & E10.0 \\
\hline $31-40$ & & Tensile Stress Cut-off Load Curve \# & E10.0 \\
\hline $41-50$ & & Compress Stress Cut-off Load Curve \# & E10.0 \\
\hline $51-60$ & & Flow Strength Model Type (2) & E10.0 \\
\hline $61-70$ & & Initial Void Strain & E10.0 \\
\hline $1-10$ & 4 & Young's Modulus for Slideline Calculations & E10.0 \\
\hline $1-10$ & 5 & Poisson Ratio for Slideline Calculations & E10.0 \\
\hline $1-10$ & 6 & Strength constant $S^{\circ}$ Load Curve \# & E10.0 \\
\hline $11-20$ & & Strength Coefficient $(A)$ Load Curve \# & E10.0 \\
\hline $21-30$ & & Strain Rate Sensitivity $(m)$ Load Curve \# & E10.0 \\
\hline $31-40$ & & Strengthening Coefficient $\left(k_{1}\right)$ Load Curve \# & E10.0 \\
\hline $41-50$ & & Strengthening Exponent $\left(n_{1}\right)$ Load Curve \# & E10.0 \\
\hline $51-60$ & & Recovery Coefficient $\left(k_{2}\right)$ Load Curve \# & E10.0 \\
\hline $61-70$ & & Recovery Exponent $\left(n_{2}\right)$ Load Curve \# & E10.0 \\
\hline $71-80$ & & Recovery Exponent $\left(n_{3}\right)$ Load Curve \# & E10.0 \\
\hline
\end{tabular}

INSERT EXAMPLE PROBLEMS HERE 


\section{FUTURE SOLIDIFICATION MODELING PLANS}

In a previous paragraph, four competing solidification modeling issues were discussed: liquid modeling, solid modeling, deviatoric and dilatational deformation behavior and liquid-solid transitional behavior. This last memo section bases the future development plans for SOLMAT on these four items. The plan is evolutionary, but the end goal is to provide a range of modeling options depending on the available material data. Already, the capabilities of SOLMAT and the material properties it requires exceed the known properties of the metals dealt with in LLNL casting operations. The last item discussed in this memo is two suggested material tests for determining the material properties useful to SOLMAT modeling.

The strain rate dependent behaviors of Models 1 and 2 predict viscoelasto-plasticity. For incompressible materials however, there should not be any elastic stress components. Even though the low stress levels make the error of this stored elastic energy small, the situation can be partially remedied by eliminating the elastic deviatoric component. A separate and distinct relationship is maintained between the deviatoric and dilatational stresses. B. Engelmann developed the idea of eliminating the elastic stress component in his work on a gas model for PALM2D. The essence of the strategy is to ignore the previous deviatoric stress state when calculating the next stress state. Thus, the deviatoric strain increment during a step is converted to a strain rate and then multiplied by the viscosity to obtain the next deviatoric stress state. The dilatational stress components are evolved with Equations 13. The incompressible "fluid" pressure equation is modeled in this "solid" framework as a compressible material with pressure constraints.

The application of a Newtonian flow theory in SOLMAT will require much testing; but B. Engelmann believes that it offers greater stability than the current practice of predicting perfect plasticity. This may be due to the fact that the stress tangent used in the solution of the Equilibrium equations is conditioned better (Figure 2). It should be noted however that artificially high viscosities are probably necessary to obtain the well conditioned tangents depicted in Figure 2. In any case, this is an important avenue of research. 


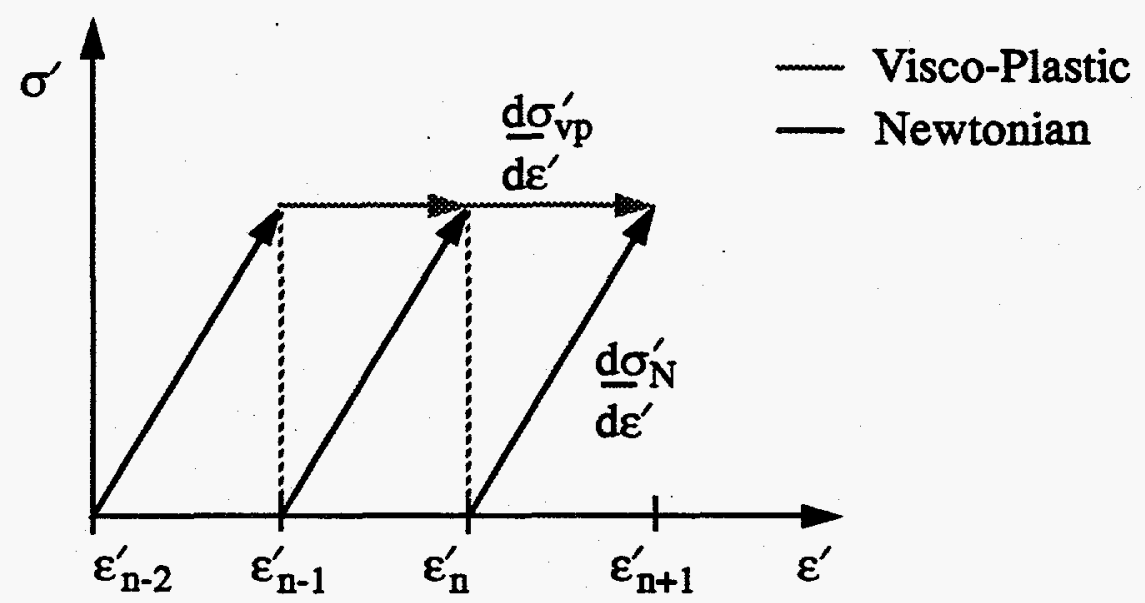

Figure 2: A comparison of visco-plastic $\sigma_{\mathrm{vp}}^{\prime}$ and Newtonian $\sigma_{\mathrm{N}}^{\prime}$ stress predictions showing the difference in stress tangents $d \sigma / d \varepsilon$.

For solid constitutive modeling improvements, aside from the dilatational - deviatoric relationship which is discussed next, the only new feature planned is a static recovery addition to the Strengthening parameter (Equation 10). This recovery term needs further research to ascertain what functional relationships is appropriate for the softness mechanism. The expected softening variables are the current Strength $S$ and temperature $T$. Time should not be a variable, since it is not a state variable, and the Strength evolves with time and not because of it.

The only other solid constitutive modeling improvement considered is the addition of a back stress variable to capture the kinematic effects of changes in loading orientations. There are compelling arguments in favor of this development, but it is not a high priority. This feature is simple to add yet requires additional modeling parameters which are difficult to quantify.

The existing cut-off stress mechanisms in SOLMAT are crude when it comes to the theoretical assumptions which underlie their implementation. Namely, SOLMAT uncouples the deviatoric and dilatational deformation behavior. This is justified for liquid behavior but is unsatisfactory for solid phase materials. For solids with voids, the dilatational and deviatoric defor- 
mation behavior is coupled through flow strength, and elastic moduli. The evolution of voids even couples to the heat transfer equations by altering the effective density, specific heat and thermal conductivity.

Constitutive models for "isotropic, porous elastic-viscoplastic metals" [1] exist in the literature. This project plans to implement the rateindependent Gurson void growth model [2], later modified by Tvergaard and Needleman [3] (GTN model). Then strain rate dependencies will be added to the GTN formulation. A Viscoplastic potential to the GTN function proposed by Haghi and Anand should also be tested since it offers superior rate predictions with one less free parameter than the GTN model. This aspect of the constitutive model development presents the largest challenge to this project. The coupling of deviatoric and dilatational flow behavior entails predicting non associative plastic flow. NIKE2D already has the GTN model (material \#25), so that facilitates its development in a temperature dependent model.

Void growth degrades elastic moduli and the thermal properties previously mentioned. Budiansky [4] derived self consistent estimates for the Bulk $K$ and Shear moduli $G$ based on their matrix (fully dense) properties. His calculations result in the following pair of equations.

$$
\begin{aligned}
& \chi_{K}=\frac{K}{K_{m}}=1-\frac{3}{2}\left(\frac{1-v}{1-2 v}\right) \varepsilon^{v} \\
& \chi_{G}=\frac{G}{G_{m}}=1-15\left(\frac{1-v}{7-5 v}\right) \varepsilon^{v}
\end{aligned}
$$

Next an elastic constitutive relationship (Equation 14) provides the third equation to solve for the three variables $\chi_{K}, \chi_{G}$ and $v$. Due to the nonlinearity of these equations, a numerical solution technique is necessary.

$$
\nu=(3 K-2 G) /(6 K+2 G)
$$


The last solidification modeling issue concerns switching between liquid and solid behaviors. This development hinges on a philosophical point of how much control the model should leave in the users hands. It also hinges on what is technically possible. What is the point of flexibility if most of the options do not work? The present SOLMAT uses a single constitutive theory for both liquid and solid modeling and depends on a temperature dependent transition in material properties. The development plan is to transition between different solid and liquid constitutive theories.

The starting idea for the problem is to linearly interpolate the stress predictions of the solid and Newtonian constitutive theories between a user supplied liquidus and solidus temperature. By setting these temperatures very high or very low a single constitutive theory model is available. The stress cut-off behavior is unchanged and the responsibility of the analyst to turn off in the solid phase.

Another aspect of the switching behavior concerns the modifications of evolutionary material variables such as $\bar{\varepsilon}, S$ and $\varepsilon^{v}$. Depending on the constitutive model type, $\bar{\varepsilon}$ and $S$ should be zeroed when solid to liquid phase changes occur. Another useful trick is to have a Melt Feed Temperature which zeros the void strain. This has a practical effect of adding mass to an element, thus melt material is fed into the element. This is an idea worth trying, because it allows the analyst to relax the CTE behavior above the Feed temperature and allow an inflow of material to supply a solidification front.

Table 4 lists the solidification modeling tasks for the next development phase. The tasks are not prioritized but the task order is likely be A-DC-B. Each development task has five associated activities: research, implementation, debugging, testing and evaluation. The first three items are the responsibility of a code developer and the last two items require an analyst. Most of the development effort is spent debugging and testing. It will take at least a man year to accomplish these tasks and another man year to correlate these constitutive theories with experimental validations. Items italicized in Table 4 are Phase III activities for another year. 
Table 4: Phase II Solidification Modeling Tasks.

\begin{tabular}{|c|l|}
\hline TASK \#. & \multicolumn{1}{|c|}{ DESCRIPTION } \\
\hline \hline A & Liquid Constitutive Modeling \\
\hline .1 & Newtonian Flow \\
\hline B & Solid Constitutive Modeling \\
\hline .1 & Static Recovery \\
\hline .2 & Kinematic Hardening \\
\hline C & Deviatoric and Dilatational Coupling \\
\hline .1 & Gurson-Tvergaard Porous Plasticity \\
\hline .2 & Self Consistent Elastic Moduli \\
\hline .3 & Gurson-Tvergaard Porous Visco Plasticity \\
\hline .4 & Haghi-Anand Porous Visco Plasticity \\
\hline .5 & Thermal Property Coupling \\
\hline D & Liquid-Solid Model Switching \\
\hline 1 & Liquid-Solid Switching \\
\hline 2 & Liquid-Solid Switching on All Constitutive Models \\
\hline 3 & Reinitialize Evolutionary Variables \\
\hline 4 & Melt Feed Temperature \\
\hline
\end{tabular}

The bottom line in getting experimental data for modeling liquidsolid deformation behavior is to get high temperature measurements. Typically there is a blackhole of information between 0.6 and 1.0 of $T_{m}$. So just about any strength measurement at these temperatures is very valuable. This 
write-up ignores tests outside this blackhole because standard tests already exist for those regimes.

The first test ought to be a simple rate dependent compression test done under an inert atmosphere. A possible test configuration is shown in Figure 3. As suggested the experiment would be performed at a variety of temperatures and strain rates. Forces and displacement measurements are correlated to contact area changes and strength evolution using numerical methods. Friction factor effects have to be factored into the strength analysis.

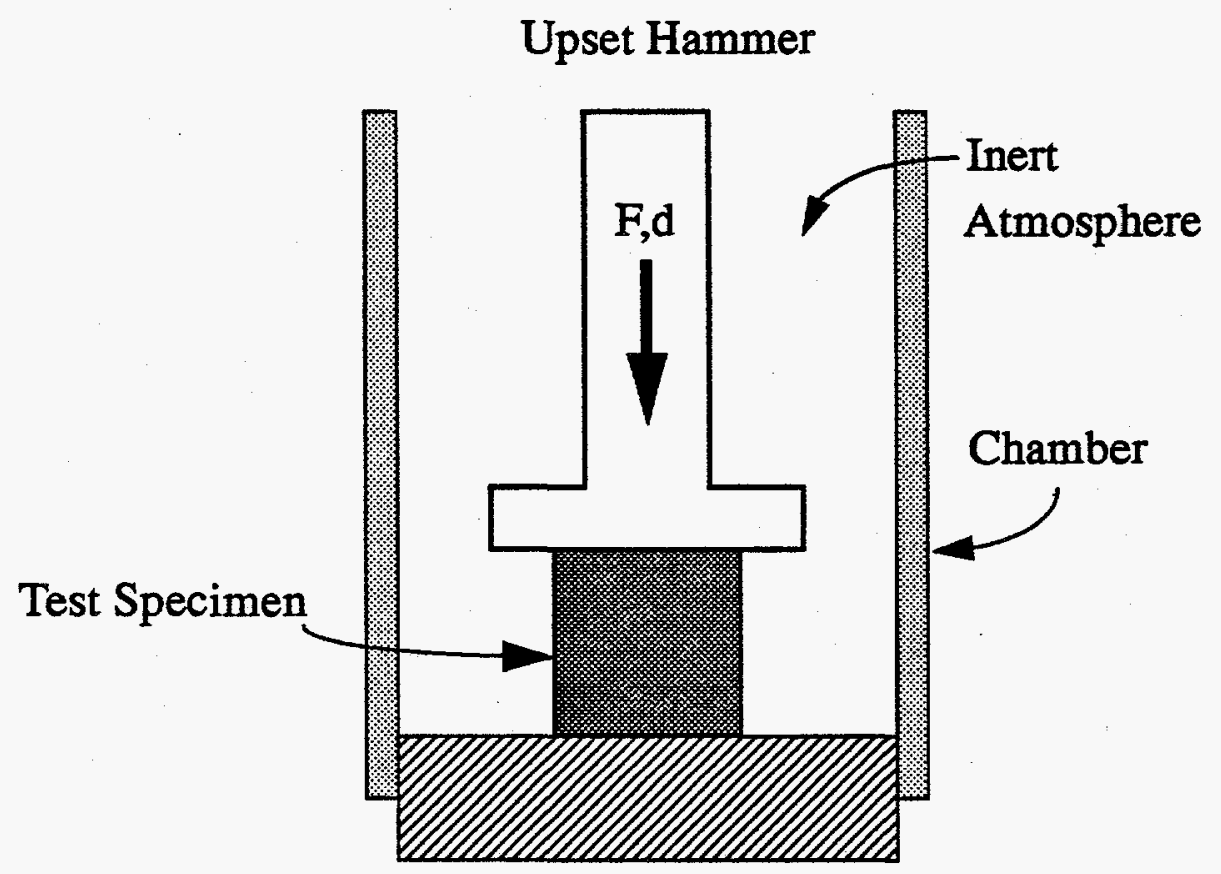

Figure 3: Compressive flow strength Experiment. 


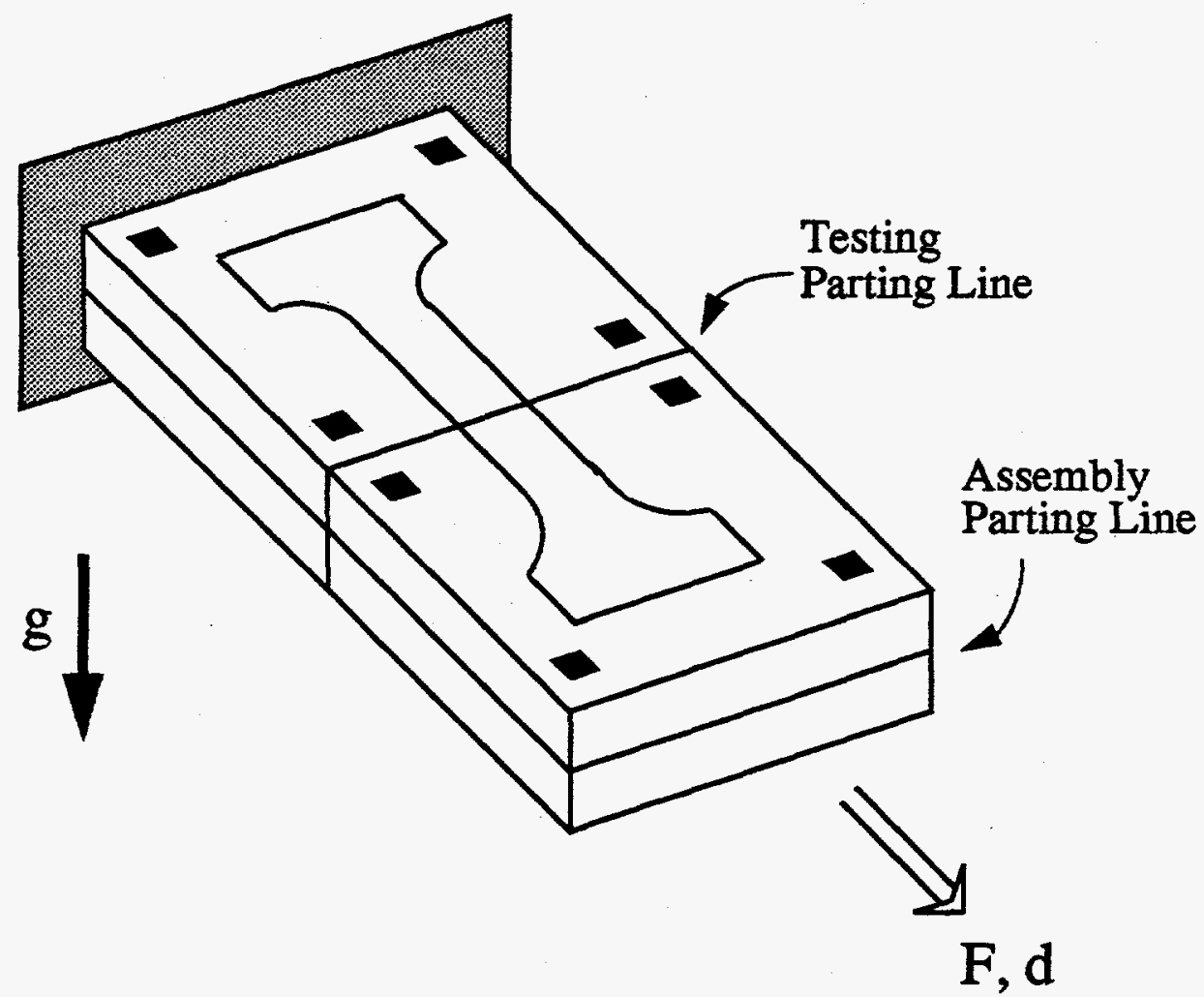

Figure 4: Tensile cut-off stress experiment

A second test to measure tensile cut-off stresses should be performed to evaluate void growth evolution laws. This experiment consists of two dies pressed together and containing a pre-machined test specimen. After melting the specimen in the horizontal dies, the specimen contacts the die walls, and solidifies during a slow cooldown to a test temperature. If the die CTE is smaller than the test material, then passive force measurements can be made during cooldown. For active measurements, the dies are separated under isothermal conditions and the displacement versus force is recorded. Multiple experiments of differing deformation extents could be performed to obtain material which can be microscopically examined to measure porosity. This data could be correlated to tensile forces and void strains.

This radical test offers a unique measurement capability which would be of a vital importance to LLNL programs which depend on very high tem- 
perature failure strength data. The test might have broad appeal, and could be a likely candidate for Engineering-Material Thrust Area funding.

\section{CONCLUSIONS}

This year the solidification material model development work succeeded in creating a solidification model called SOLMAT which has been implemented in NIKE2D, NIKE3D and NITO3D. SOLMAT uses tensile and compressive stress cut-off mechanisms to avoid unrealistic hydrostatic pressures in the liquid phase. This corrects some earlier results and adds confidence to the solidification deformation predictions. SOLMAT is capable of predicting both elasto-plastic and elasto-viscoplastic deformation. In addition, dynamic recovery effects are included in the model which expands the applications of this model to welding simulations.

The future development plans for SOLMAT include improvements to liquid deformation with Newtonian flow. Solid deformation can be enhanced with static recovery and kinematic hardening. The most ambitious aspect of this work concerns the coupling of deviatoric and dilatational deformation using Gurson void growth equations. All these developments need integration in SOLMAT as solidification switches from liquid to solid deformation mechanisms. Finally two experimental suggestions are offered to supply data for SOLMAT. 


\section{REFERENCES}

[1] M. Haghi and L. Anand, "A Constitutive Model for Isotropic, Porous Elastic-Viscoplastic Metals," Mechanics of Materials 13 (1992) pp. 37-53.

[2] A.L. Gurson, "Continuum Theory of Ductile Rupture by Void Nucleation and Growth: Part 1 - Yield Criteria and Flow Rules for Porous Ductile Media," Trans. ASME J. Eng. Mater. Technol., 99, 2, 1977.

[3] V. Tvergaard, "Material Failure by Void Growth to Coalescence," Adv. Appl. Mech., 27, 83, 1989.

[4] B. Budiansky, "Thermal and Thermoelastic Properties of Isotropic Composites," J. Composite. Mater. 4 p. 286, 1970.

cc B. Comfort, B. Engelmann, G. Goudreau, D. Schauer. 